\title{
CHARACTERIZATION OF TRAFFIC-RELATED PARTICULATE MATTER AT URBAN SCALE
}

\author{
D. DIAS, N. PINA \& O. TCHEPEL \\ CITTA and Department of Civil Engineering, University of Coimbra, Polo II, 3030-788 \\ Coimbra, Portugal
}

\begin{abstract}
Road traffic is recognized as a significant source of particulate matter (PM), especially in urban areas, where exceedances of the legislation PM limit values is one of the main environmental concerns. Therefore, the development and implementation of methodologies allowing detailed characterization of PM within urban areas are required to find potential solutions to decrease PM levels.

This work aims to provide a detailed characterization of traffic-related PM concentrations at urban scale by using an integrated modelling approach and in-situ aerosol measurements. For this purpose, a modelling cascade based on transportation-emission-dispersion approach was implemented for a medium-sized Portuguese city (Coimbra). Moreover, optical aerosol measurements were obtained from an experimental field monitoring campaign (June 2017) implemented at a city 'hot-spot' to provide relevant in-situ data on number, surface and mass concentrations distribution into 31 size ranges from 0.25 to $32 \mu \mathrm{m}$.

The spatial distribution of the exhaust and non-exhaust traffic-related emissions is analysed and discussed addressing their contribution to the PM pollution. The current study evidences the importance of road traffic non-exhaust emissions and demonstrates the usefulness of the integrated modelling approach in the mobility policy relevant context.

Keywords: aerosol, non-exhaust emissions, particulate matter, road transport and urban area
\end{abstract}

\section{INTRODUCTION}

Road transport remains an important source for some of the most harmful air pollutants. In particular, road transport is responsible for significant contributions to emissions of particulate matter (PM), especially in urban environments and major cities. Despite slow improvements, PM concentrations continue to exceed European Union and World Health Organization limits and guidelines in large parts of Europe [1]. Potential impacts of air pollution on human health are investigated in several studies [2-5]. It is demonstrated that in European countries, the exposure to $\mathrm{PM}_{2.5}$ concentrations reduces the average life expectancy by 8.6 months [2]. Additionally, Analitis et al. [3] found out from an investigation in 29 European countries, that mortality due to respiratory problems increased by $0.58 \%(0.21-0.95 \%)$ for every $10 \mu \mathrm{g} \mathrm{m}^{-3}$ increase of $\mathrm{PM}_{10}$. Moreover, it is expected that the air pollution contribution to global premature mortality could double by 2050 [4]. Consequently, the air pollution remains the single largest environmental health risk in Europe [6], particularly in urban areas [7].

In addition to exhaust traffic-related PM emissions, the non-exhaust part of emissions such as those from brake wear, road wear, tyre wear and road dust resuspension have a large contribution to the atmospheric PM concentrations in cities. This contribution may be even larger than those from the exhaust sources, while due to continuous reduction of exhaust emissions, it is expected that the relative contribution of non-exhaust sources will increase in the forthcoming years [8]. Despite the importance of non-exhaust traffic-related emissions, scientific knowledge on this source of PM is scarce, thus limiting the correct implementation of mitigation strategies to control the high atmospheric PM levels observed in cities. 
Given the need to better inform policy-makers in the implementation and evaluation of cost-effective transport policies, it is essential to develop and implement methodologies to provide reliable and spatially resolved PM estimates within cities. However, estimating the temporal and spatial variabilities of traffic-related PM emissions and their concentrations at urban scale is challenging.

The aim of this study is to provide a comprehensive characterization of traffic-related PM spatial and temporal variation at urban scale, by using an integrated modelling approach and in-situ aerosol measurements. For this purpose, a modelling cascade based on transportation-emission-dispersion modelling was implemented for the urban area of Coimbra. The emission model has been extended to include a new module for quantifying emissions of different metrics of PM released to atmosphere from non-exhaust traffic-related sources, namely brake, tyre and road surface wear with detailed spatial discretization. These emission data will be one of the most important inputs to the urban dispersion modelling. Moreover, optical aerosol measurements, obtained during an experimental field monitoring campaign implemented at a city 'hot-spot', are considered for the model validation.

\section{METHODOLOGY}

The Portuguese city of Coimbra was selected in this study to estimate and measure trafficrelated air pollution (Fig. 1). This medium-size city, with approximately 150,000 inhabitants, is the largest urban area in central Portugal. In-situ measurements campaign design and the integrated modelling system applied in this study are described in the following sections.

\subsection{In-situ measurements}

An experimental field monitoring campaign took place in the central area of Coimbra, close to historic centre, during 5 days in June (20-24 June 2017). During this period, several parameters were collected. For characterizing PM levels, the distribution of PM in 31 size range from 0.25 to $32 \mu \mathrm{m}$ was acquired by the GRIMM portable aerosol spectrometer (1-min resolution) [9]. Meteorological conditions including wind speed and direction, temperature,

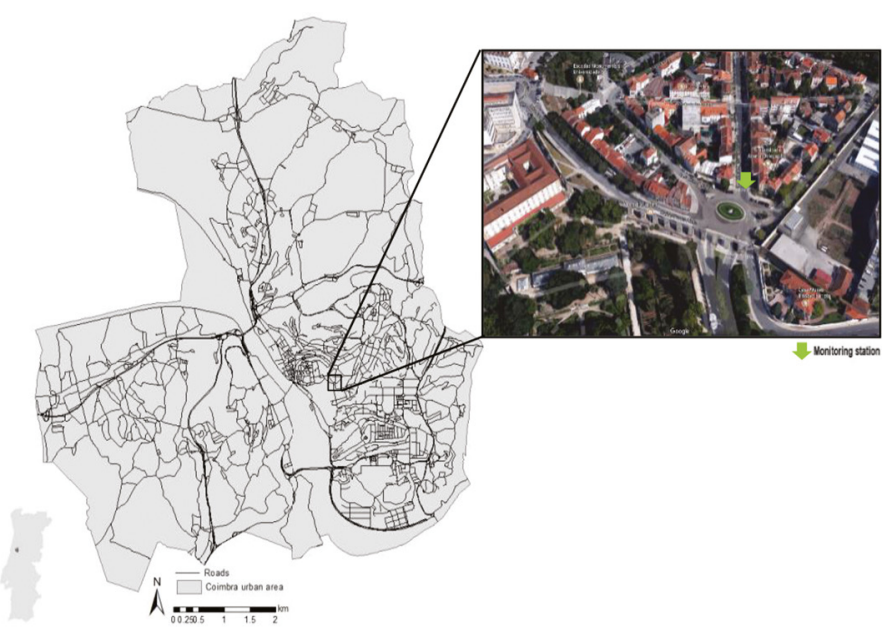

Figure 1: Study domain including road network and in-situ measurements campaign location. 
precipitation and relative humidity were measured by a mobile meteorological station. For the traffic volume characterization, eight counting point were used, providing information on temporal variation of traffic distinguished between four vehicle categories, including passenger cars, light duty vehicles, buses and motorcycles.

\subsection{Integrated modelling approach}

The proposed modelling approach to evaluate traffic-related PM concentrations consists of three interconnected models: a transportation model, an emissions model and an air quality model (Fig. 2).

Firstly, a macroscopic transportation model VISUM [10] was applied to quantify transport activity data. Thus, based on the 'four-steps' traffic modelling approach, data related with traffic volume and vehicle speed for each road segment were estimated by VISUM. Information on origin, destination, trip duration, route taken, average speed, road length and width of the city network was fully characterized. The outputs of this model were used as inputs by the emission model to quantify the emission amounts with high temporal and spatial resolution.

The Traffic Emission and Energy Consumption Model (QTraffic) was applied to Coimbra urban area in order to quantify PM emissions induced by road traffic from exhaust and non-exhaust sources. This model is based on the updated European guidelines, following an average-speed approach, and is particularly designed for line sources, estimating emissions individually for each road segment based on emission factors determined according to average speed and vehicle class (based on engine age, type and capacity, vehicle weight, fuel type and emission reduction technology). Thus, the input data required by the emissions model have been compiled on the basis of statistical information on fleet composition, spatial data on road network, and traffic volume and vehicle speed from the VISUM model. The detailed emission data are considered as important inputs to simulate air pollution dispersion at urban scale.

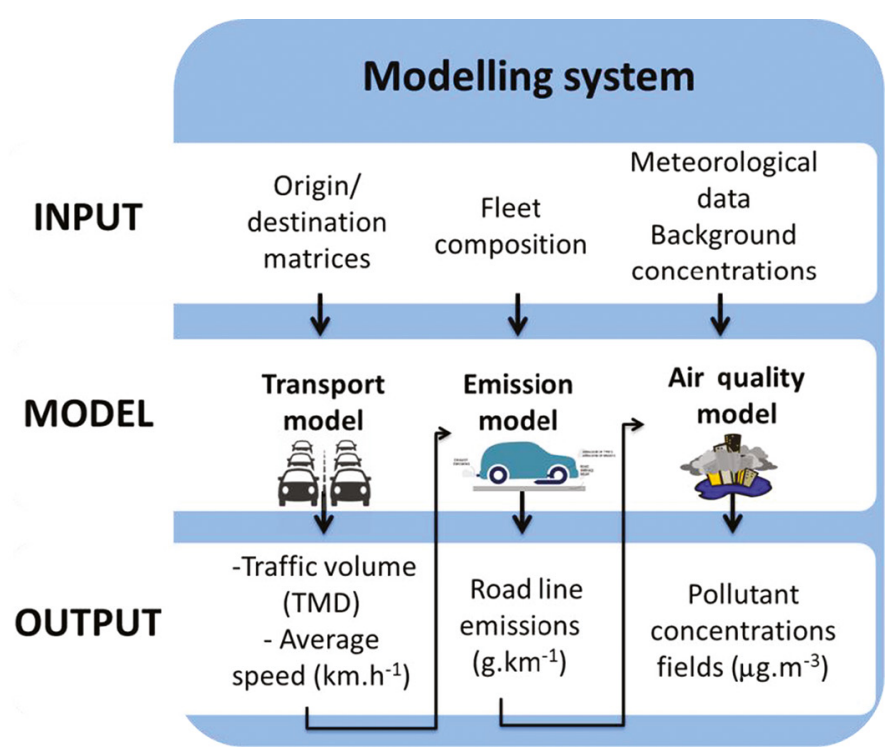

Figure 2: Integrated modelling system implemented for Coimbra urban area. 
In this work, a version of the Atmospheric Dispersion Modelling System adapted for line sources (ADMS-Roads) is used. This model is based on the Gaussian approach enabling establishment of relationship between emissions and air quality at urban scale [11]. Additionally to the emission data, a continuous time series of meteorological parameters (including wind direction, wind speed and cloudiness) are required for the model application. The results from ADMS-Road are analysed in terms of spatial distribution of pollutant concentrations with 1-h resolution.

\section{CHARACTERIZATION OF TRAFFIC-RELATED PM AT URBAN SCALE}

The characterization of traffic-related PM at urban scale by using an integrated modelling approach and in-situ aerosol measurements is presented and discussed in this section.

\subsection{Local measurements results}

The measured data were analysed for 5 days in June (20-24 June 2017). It should be noted that there have been forest fires during this period (until 22nd June) in the surrounding municipalities, as indicated in Fig. 3.

The temporal variation of data acquired by the GRIMM portable aerosol spectrometer (1-min resolution), distributed in 31 size range from 0.25 to $32 \mu \mathrm{m}$, is evidenced in Fig. 3. The influence of forest fires at the beginning of the campaign is reflected, mainly to the finest PM fraction $(0.25-0.28 \mu \mathrm{m})$. After June 22, the temporal variation of the number of particles tends to follow the daily profile of road traffic sources.

Analysing the PM size distribution in terms of mass (Fig. 4a) and surface area (Fig. 4b) is possible to verify that the contribution of finest fraction $(<0.5 \mu \mathrm{m})$ is more evident in terms of PM surface area in comparison to PM mass size distribution, mainly in the presence of forest fires (June 21). After the forest fires (June 23) the contribution of fine particles continues to be relevant, revealing a strong contribution of the anthropogenic sources to atmospheric PM levels measured in the study area.

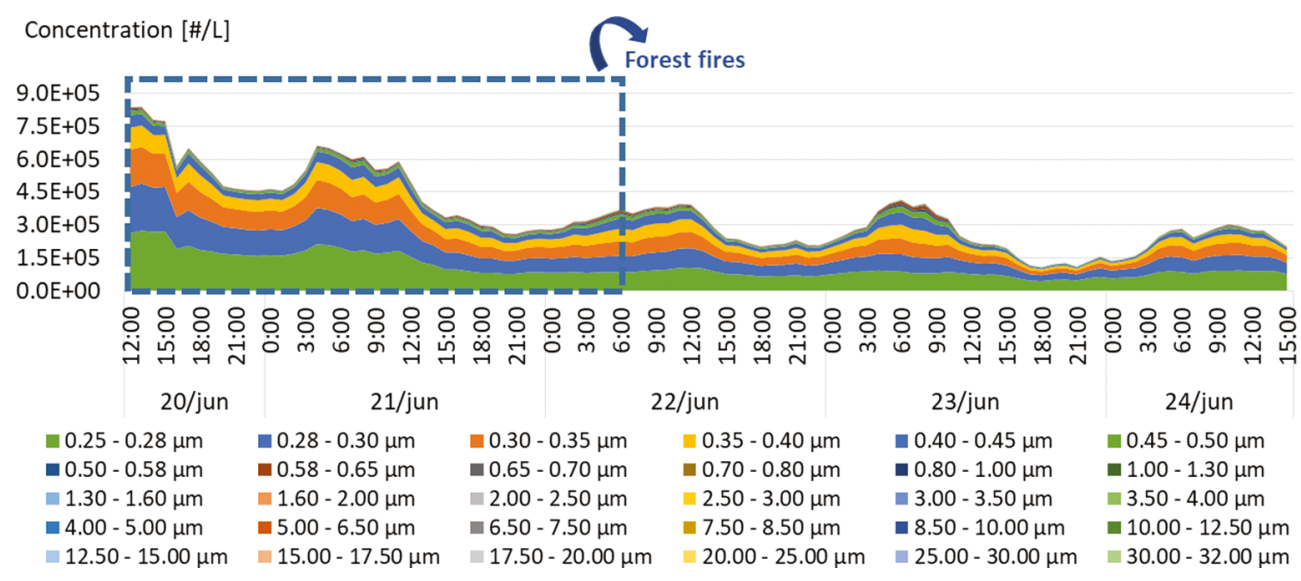

Figure 3: Temporal variation of particle number concentrations obtained from GRIMM. 


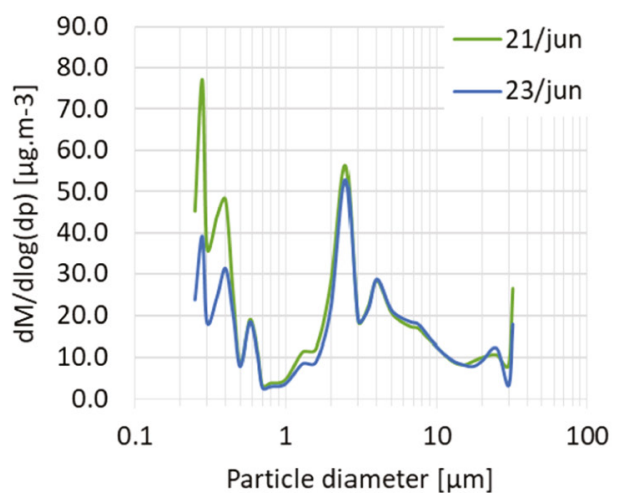

(a)

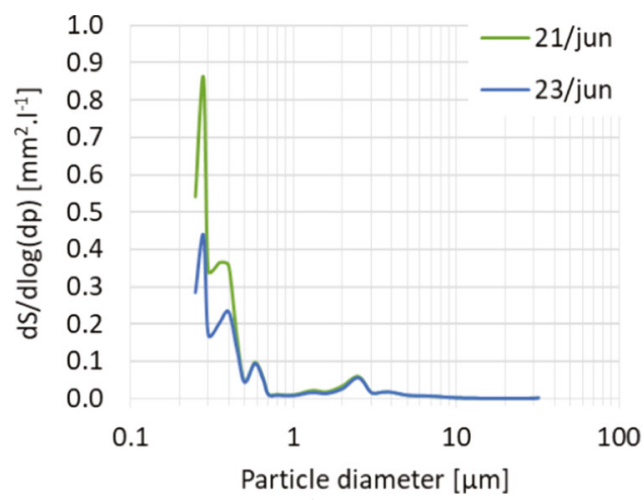

(b)

Figure 4: Distribution of hourly measurements of: (a) PM mass and (b) PM surface area obtained by GRIMM for 21st June and 23rd June.

\subsection{Integrated modelling results}

The results presented in the following figures are obtained by the QTraffic model (PM emissions) and the ADMS model (PM concentrations).

Figure 5a illustrates, as an example, the daily $\mathrm{PM}_{10}$ emissions at each road in a typical working day. As shown in Fig. 5a, higher pollutant amount emitted by road transport to the atmosphere is observed for main city entrances as well as urban roads. Moreover, the emission modelling results are analysed in terms of individual contribution of non-exhaust sources to the total PM emissions estimated (Fig. 5b). The outputs of the QTraffic indicate

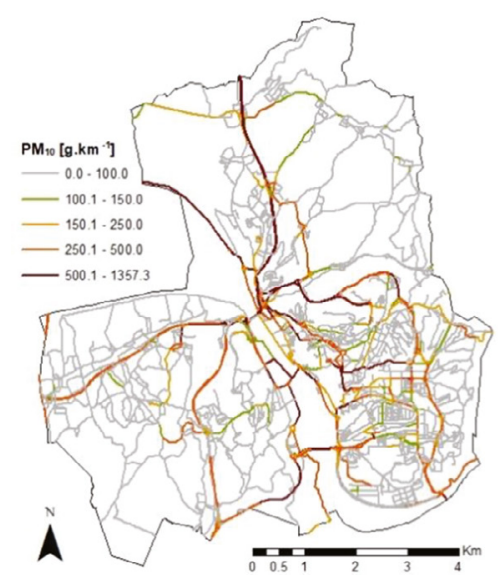

(a)

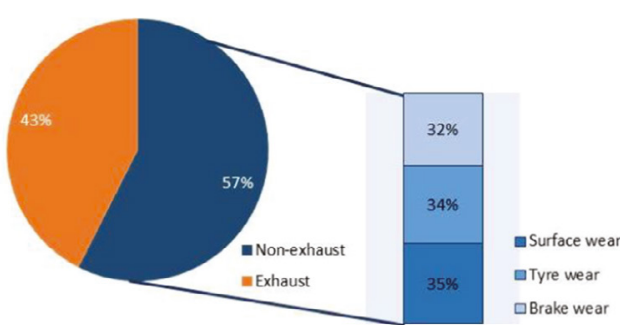

(b)

Figure 5: (a) Spatial distribution of total daily $\mathrm{PM}_{10}$ emissions within the study domain; and (b) source contribution to $\mathrm{PM}_{10}$ emissions. 


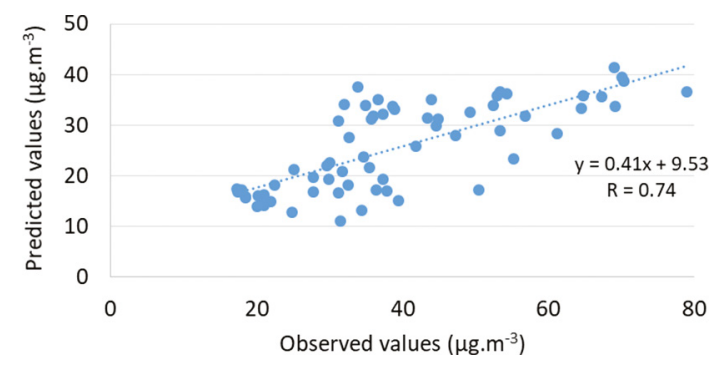

Figure 6: Scatter plot of $\mathrm{PM}_{10}$ hourly concentrations from 22nd to 24th June based on total emissions (exhaust and non-exhaust).

that in Coimbra urban area the non-exhaust emissions are more than half to the total traffic $\mathrm{PM}_{10}$ emissions, being practically equally attributed to brake, tyre and road surface wear.

Hourly and daily PM concentrations were estimated based on two traffic emission setups: (1) exhaust emissions only and (2) total (exhaust + non-exhaust) emissions. The PM hourly concentrations were compared with the PM observations from 22nd to 24th June. Figure 6 presents the scatter plot for the hourly concentrations resulting from total traffic emissions, where is seen that the correlation between the model outputs and observations is 0.74 . The good performance of the ADMS model is evidenced by another statistical parameters such as the fraction of predictions within a factor of two of observations (FAC2 $=0.9$ ), the mean normalised bias $(\mathrm{MNB}=-0.35)$ and the normalised mean square error $(\mathrm{NMSE}=0.3)$. The comparison of two modelling runs shows that non-exhaust emissions are very important resulting in a maximum difference of $4.8 \mu \mathrm{g} \mathrm{m}^{-3} \mathrm{PM}$ hourly concentration at the observation point on 23rd June at 9 AM. Overall, the model is underestimating PM concentrations and the most significant differences are obtained for nocturnal period that is not affected by traffic emissions.

Figure 7 illustrates the spatial distribution of the $\mathrm{PM}_{10}$ daily mean concentrations for a selected day (23rd June, day without occurrence of forest fires). In the map, higher concentrations in the centre of the study domain (Fig. 7a) is shown, where several activities occurs

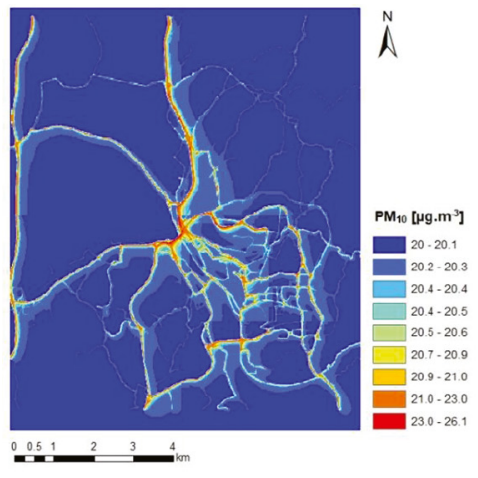

(a)

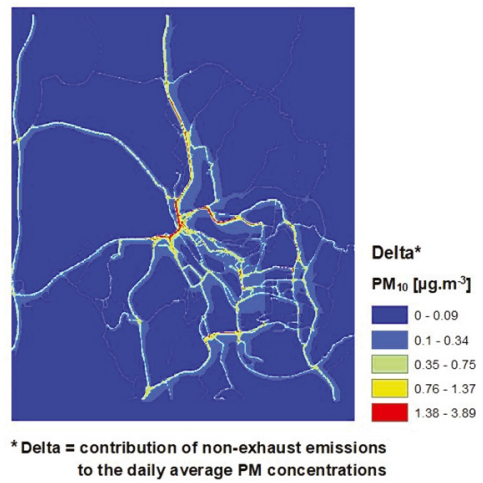

(b)

Figure 7: Spatial distribution of $\mathrm{PM}_{10}$ daily average concentrations within the study domain for 23rd June: (a) based on total traffic emissions (exhaust and non-exhaust) and (b) contribution of non-exhaust emissions. 
during the day and, therefore, people may be more exposed to harmful $\mathrm{PM}_{10}$ concentrations. Figure $7 \mathrm{~b}$ presents a difference between the two model runs thus highlighting the contribution of non-exhaust traffic emissions that achieve $4 \mu \mathrm{g} \mathrm{m}^{-3}$ in terms of daily average concentration, and about $9 \mu \mathrm{g} \mathrm{m}^{-3}$ in terms of hourly concentration at $9 \mathrm{AM}$. Therefore, the inclusion of these traffic emissions is very important as it enable to improve the model performance and identify the hotspots within the study domain.

\section{CONCLUSIONS}

In this study, a detailed characterization of traffic-related PM pollution at urban scale was performed by using an integrated modelling approach and in-situ aerosol measurements.

The in-situ measurements indicates a higher presence of fine particles in the first days of the field campaign, which is not justified by road traffic only and influenced by other sources such as forest fires. Furthermore, the study presents relevant data of PM distribution in 31 size range, which may be important for source apportionment and population exposure studies.

In terms of integrated modelling approach, the inclusion of non-exhaust emissions increases the $\mathrm{PM}_{10}$ hourly concentrations by $9 \mu \mathrm{g} \mathrm{m}^{-3}$, improving the modelling performance. Moreover, the application of the methodology enabled to identify some hotspots in the urban area by analysing the spatial distribution of the PM concentrations.

To guarantee the completeness of the emission inventory, cold-start traffic emissions and PM resuspension should be considered for future research.

\section{ACKNOWLEDGEMENTS}

The authors wish to thank to the financial support of the TRAPHIC project (PTDC/ECMURB/3329/2014 - POCI-01-0145-FEDER-016729), the ISY-AIR project (MIT-EXPL/ IRA/0023/2017) and of the Foundation for Science and Technology for the Ph.D. grant of N. Pina (PD/BD/128048/2016).

\section{REFERENCES}

[1] European Environment Agency, Air quality in Europe - 2018 report. EEA Report No. 12/2018, 2018.

[2] Orru, H., Maasikmets, M., Lai, T., Tamm, T., Kaasik, M., Kimmel, V., Orru, K., Merisalu, E. \& Forsberg, B., Health impacts of particulate matter in five major Estonian towns: main sources of exposure and local differences. Air Quality, Atmosphere and Health, 4(3), pp. 247-258, 2011.

[3] Analitis, A., Katsouyanni, K., Dimakopoulou, K., Samoli, E., Nikoloulopoulos, A. K., Petasakis, Y., Touloumi, G., Schwartz, J., Anderson, H. R., Cambra, K., Forastiere, F., Zmirou, D., Vonk, J. M., Clancy, L., Kriz, B., Bobvos, J. \& Pekkanen, J., Short-term effects of ambient particles on cardiovascular and respiratory mortality. Epidemiology, 17(2), pp. 230-233, 2006.

[4] Lelieveld, J., Evans, J. S., Fnais, M., Giannadaki, D. \& Pozzer, A., The contribution of outdoor air pollution sources to premature mortality on a global scale. Nature, 525(7569), pp. 367-371, 2015.

[5] Tchepel, O. \& Dias, D., Quantification of health benefits related with reduction of atmospheric PM10 levels: implementation of population mobility approach. International Journal of Environmental Health Research, 21(3), pp. 189-200, 2011.

[6] World Health Organization, Public Health, Environmental and Social Determinants of Health (PHE) e-News, 2017. 
[7] Dias, D. \& Tchepel, O., Spatial and temporal dynamics in air pollution exposure assessment. International Journal of Environmental Research and Public Health, 15(3), pp. 558, 2018.

[8] Amato, F., Cassee, F. R., van der Gon, H. A. D., Gehrig, R., Gustafsson, M., Hafner, W., Harrison, R. M., Jozwicka, M., Kelly, F. J., Moreno, T., Prevot, A. S. H., Schaap, M., Sunyer, J. \& Querol, X., Urban air quality: the challenge of traffic non-exhaust emissions. Journal of Hazardous Materials, 275, pp. 31-36, 2014.

[9] GRIMM Aerosol Technik GmbH \& Co. KG, Portable Laser Aerosol Spectrometer Model 11-C manual. Ainring, Germany, 2015.

[10] PTV, VISUM 13 User Guide. PTV-AG, Karlsruhe, Germany, 2013.

[11] CERC, ADMS-Roads, An Air Quality Management System User Guide. v. 4.1, Cambridge Environmental Research Consultants, Cambridge, UK., 2017. 